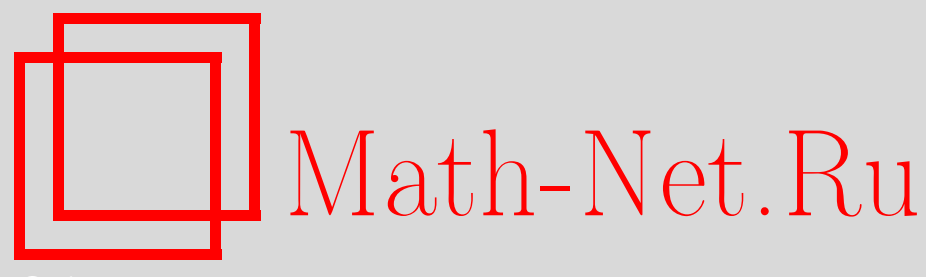

Ли-Янь Лю, Цин-Хай Хао, Плоский водородоподобный атом в неоднородных магнитных полях: точно или квазиточно решаемые модели, ТМФ, 2015, том 183, номер 2, 329-336

DOI: https://doi.org/10.4213/tmf8707

Использование Общероссийского математического портала Math-Net.Ru подразумевает, что вы прочитали и согласны с пользовательским соглашением http: //www . mathnet.ru/rus/agreement

Параметры загрузки:

IP: 34.239 .49 .27

26 апреля 2023 г., 13:03:34

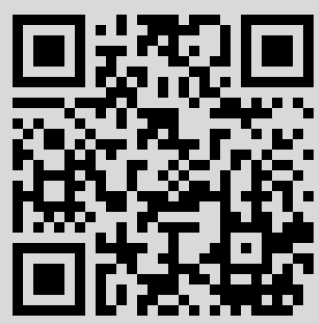




\title{
ПЛОСКИЙ ВОДОРОДОПОДОБНЫЙ АТОМ В НЕОДНОРОДНЫХ МАГНИТНЫХ ПОЛЯХ: ТОЧНО ИЛИ КВАЗИТОЧНО РЕШАЕМЫЕ МОДЕЛИ
}

\begin{abstract}
Для решения задачи о движении двумерного водородоподобного атома в неоднородных магнитных полях вида $\mathbf{B}=(k / r) \mathbf{z}$ и $\mathbf{B}=\left(k / r^{3}\right) \mathbf{z}$ применяется простой математический метод. Построен гамильтониан, имеющий тот же вид, что и гамильтониан водородоподобного атома в однородных магнитных полях, и с помощью сравнения гамильтонианов получен энергетический спектр. Результаты показали, что можно получить весь спектр атома в магнитном поле $\mathbf{B}=(k / r) \mathbf{z}$, таким образом, в этом случае задача является точно решаемой. Найдены аналитические решения уравнения Шредингера для атома в магнитном поле $\mathbf{B}=\left(k / r^{3}\right) \mathbf{z}$ при определенных значениях магнитной напряженности $k$, тем самым представлена квазиточно решаемая модель.
\end{abstract}

Ключевые слова: квазиточно решаемая система, точно решаемая система.

DOI: $10.4213 / \operatorname{tmf} 8707$

\section{1. ВВЕДЕНИЕ}

В квантовой механике очень важны точные решения уравнения Шредингера, так как в них содержится вся необходимая информация о рассматриваемой системе [1], [2]. В общем случае уравнение Шредингера нельзя решить точно и получить полный спектр. Для большинства потенциалов спектр можно рассчитать только численно. Недавно было обнаружено, что для некоторых квантовых задач часть собственных значений и собственных функций (но не весь спектр) может быть получена аналитически, такие задачи называются квазиточно решаемыми [3]-[9]. Квазиточно решаемые задачи занимают важное место в квантовой механике, и физические примеры таких систем привлекают много внимания [10]-[15].

Плоское движение электронов во внешних электромагнитных полях вызывает большой интерес вследствие их классического поведения и наличия богатых квантовых структур [16]-[19]. Недавно было обнаружено, что для двумерного атома водорода, движущегося в однородном магнитном поле, можно получить аналитические решения уравнения Шредингера [20]-[22], уравнения Клейна-Гордона [23]

${ }^{*}$ College of Science, Civil Aviation University of China, Tianjin, China.

E-mail: llypaper@126.com 
и уравнения Дирака [24]-[26], и все эти уравнения являются физическими примерами квазиточно решаемых задач. В предыдущих исследованиях атома водорода во внешних полях в основном рассматривались однородные магнитные поля, а их свойства в неоднородных полях не обсуждались.

В настоящей статье мы изучаем водородоподобный атом в магнитных полях вида $\mathbf{B}=(k / r) \mathbf{z}$ и $\mathbf{B}=\left(k / r^{3}\right) \mathbf{z}$, где $k$ - константа, $r$ - полярная координата и $\mathbf{z}-$ единичный вектор в направлении оси $z$. Мы не обсуждаем механизм возникновения неоднородных магнитных полей, вместо этого мы фокусируем наше внимание на физических свойствах атомов в них и демонстрируем новые физические модели точно и квазиточно решаемых задач.

Статья построена следующим образом. В разделе 2 изучается задача о плоском водородоподобном атоме, движущемся в магнитном поле $\mathbf{B}=(k / r) \mathbf{z}$. Вместо того чтобы непосредственно решать уравнение Шредингера, мы представляем новый способ получения энергетического спектра. В разделе 3 мы применяем этот метод, чтобы рассмотреть движение атома в магнитном поле $\mathbf{B}=\left(k / r^{3}\right) \mathbf{z}$. Показано, что для некоторых значений напряженности магнитного поля $k$ можно получить аналитические решения, следовательно, мы имеем физическую модель квазиточно решаемой задачи. Наконец, итоги и обсуждение даны в разделе 4.

\section{2. АТОМ В МАГНИТНОМ ПОЛЕ $\mathbf{B}=(k / r) \mathbf{z}$}

Пусть магнитное поле $\mathbf{B}=(k / r) \mathbf{z}$ перпендикулярно плоскости, в которой расположен атом. Векторные потенциалы, связанные с магнитным полем, выраженные в полярных координатах $(r, \theta)$ записываются как

$$
A_{x}=-k \sin \theta, \quad A_{y}=k \cos \theta, \quad A_{z}=0,
$$

где применяется кулоновская калибровка. В настоящей работе мы используем атомные единицы $M=e=\hbar=1$. Гамильтониан водородоподобного атома в магнитном поле имеют вид [1], [2]

$$
H_{1}=\frac{1}{2}\left(\mathbf{p}+\frac{\mathbf{A}}{c}\right)^{2}-\frac{Z}{r}
$$

где $c$ - скорость света и $Z$ - заряд атома. Мы вводим собственную функцию следующего вида:

$$
\Psi(r, \theta)=\frac{U(r)}{\sqrt{r}} e^{i m \theta},
$$

где $m$ - квантовое число углового момента, являющееся целым числом. Подставляя эту собственную функцию в уравнение Шредингера

$$
H_{1} \Psi(r, \theta)=E_{1} \Psi(r, \theta),
$$

мы получаем, что радиальная функция $U(r)$ удовлетворяат дифференциальному уравнению второго порядка

$$
\frac{1}{2}\left(-\frac{d^{2}}{d r^{2}}+\frac{m^{2}-1 / 4}{r^{2}}+\frac{2 K m-2 Z}{r}+K^{2}\right) U(r)=E_{1} U(r),
$$

где $K=k / c$. Это уравнение можно переписать в виде

$$
H_{1 r} U(r)=E_{1} U(r),
$$


где

$$
H_{1 r}=\frac{1}{2}\left(-\frac{d^{2}}{d r^{2}}+\frac{m^{2}-1 / 4}{r^{2}}+\frac{2 K m-2 Z}{r}+K^{2}\right) .
$$

Мы не будем решать уравнение (2) непосредственно, вместо этого мы построим гамильтониан, который имеет такой же вид, как и $H_{1 r}$, и, сравнивая гамильтонианы, получим энергетический спектр.

Мы построим гамильтониан вида

$$
H_{1}^{\mathrm{c}}=\frac{1}{2}\left(-\frac{d^{2}}{d x^{2}}+W^{2}-\frac{d W}{d x}+V(x)\right)
$$

где индекс с отвечает сконструированному гамильтониану. Функция $W(x)$ дается выражением

$$
W(x)=a+\frac{b}{x}, \quad x \geqslant 0,
$$

где $a, b$ - константы. В $(4) V(x)$ - потенциал, который надо определить. Мы считаем, что собственные функции гамильтониана $H_{1}^{\mathrm{c}}$ имеют вид

$$
\psi_{N}^{\mathrm{c}}(x)=\sum_{i=0}^{N} a_{i} x^{i} \exp \left(-\int W(x) d x\right)=\sum_{i=0}^{N} a_{i} x^{i} x^{-b} e^{-a x},
$$

где $a_{i}$ - коэффициенты разложения. Чтобы волновая функция оставалась конечной в пределах $x \rightarrow 0$ и $x \rightarrow \infty$, мы считаем, что $b<0$ и $a>0$. Подстановка $\psi_{N}^{\text {c }}(x)$ в уравнение Шредингера

$$
H_{1}^{\mathrm{c}} \psi_{N}^{\mathrm{c}}(x)=E_{1}^{\mathrm{c}} \psi_{N}^{\mathrm{c}}(x)
$$

дает

$$
\begin{aligned}
& V(x)-2 E_{1}^{\mathrm{c}}=-\frac{2 b a_{1}}{a_{0} x}+ \\
& +\frac{\sum_{i=2}^{N} a_{i} i(i-1) x^{i-2}-2 b \sum_{i=2}^{N} a_{i} i x^{i-2}-2 a \sum_{i=2}^{N} a_{i} i x^{i-1}+\left(2 b a_{1} / a_{0}\right) \sum_{i=1}^{N} a_{i} x^{i-1}}{\sum_{i=0}^{N} a_{i} x^{i}} .
\end{aligned}
$$

Пусть

$$
V(x)=-\frac{2 b a_{1}}{a_{0} x}
$$

и

$$
\begin{aligned}
& -2 E_{1}^{\mathrm{c}}= \\
& =\frac{\sum_{i=2}^{N} a_{i} i(i-1) x^{i-2}-2 b \sum_{i=2}^{N} a_{i} i x^{i-2}-2 a \sum_{i=2}^{N} a_{i} i x^{i-1}+\left(2 b a_{1} / a_{0}\right) \sum_{i=1}^{N} a_{i} x^{i-1}}{\sum_{i=0}^{N} a_{i} x^{i}} .
\end{aligned}
$$

Дробь в правой части последнего равенства следует приравнять константе, чтобы уравнение оставалось верным, а если ее можно свести к константе, то она равна нулю:

$$
\sum_{i=2}^{N} a_{i} i(i-1) x^{i-2}-2 b \sum_{i=2}^{N} a_{i} i x^{i-2}-2 a \sum_{i=2}^{N} a_{i} i x^{i-1}+\frac{2 b a_{1}}{a_{0}} \sum_{i=1}^{N} a_{i} x^{i-1}=0 .
$$


Из этого соотношения получаются рекуррентные формулы для коэффициентов $a_{i}$ :

$$
a_{i+1}=\frac{2 a(i-N)}{(i+1)(i-2 b)} a_{i} .
$$

Отсюда имеем

$$
V(x)=-\frac{2 a N}{x}, \quad E_{1}^{\mathrm{c}}=0 .
$$

Таким образом, $H_{1}^{c}$ записывается как

$$
H_{1}^{\mathrm{c}}=-\frac{1}{2} \frac{d^{2}}{d x^{2}}+\frac{1}{2}\left(\frac{b^{2}+b}{x^{2}}+\frac{2 a b-2 a N}{x}+a^{2}\right) .
$$

Если мы положим

$$
x=r, \quad b^{2}+b=m^{2}-\frac{1}{4}, \quad a b-a N=K m-Z,
$$

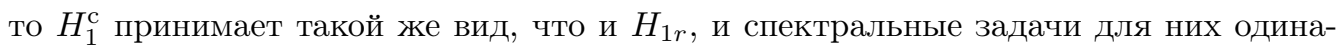
ковы. Из соотношений (7) легко получить равенства

$$
b=-|m|-\frac{1}{2}, \quad a=\frac{K m-Z}{-|m|-1 / 2-N} .
$$

Чтобы удовлетворить неравенство $a>0$, необходимо наложить на $m$ некоторые ограничения: если магнитное поле направлено вдоль положительной части оси $z$, а именно, $K>0$, то $m<Z / K$; если магнитное поле направлено вдоль отрицательной части оси $z$, т. е. $K<0$, то $m>Z / K$. Отсюда для водородоподобного атома, движущегося в магнитном поле $\mathbf{B}=(k / r) \mathbf{z}$, квантовые числа углового момента не могут быть произвольными целыми числами, ограничения для них определяются напряженностью магнитного поля и атомным зарядом, в то время как в однородном магнитном поле таких ограничений нет, и $m$ может быть произвольным целым числом [21]. Сравнивая формулы (3), (6) и (8), мы получаем весь энергетический спектр водородоподобного атома:

$$
\begin{aligned}
E_{m n} & =\frac{1}{2}\left[K^{2}-\left(\frac{Z-K m}{n}\right)^{2}\right] \\
\Psi_{m n}(r, \theta) & =\sum_{i=0}^{n-|m|-1 / 2} a_{i} r^{i+|m|} \exp \left(\frac{K m-Z}{n} r\right) e^{i m \theta}, \quad n=|m|+N+\frac{1}{2},
\end{aligned}
$$

где

$$
a_{i+1}=\frac{K m-Z}{-|m|-1 / 2-N} \frac{(2 i-2 n+2|m|+1)}{(i+1)(i+2|m|+1)} a_{i} .
$$

Видно, что энергия связана с напряженностью магнитного поля, и ее вырождение снимается с помощью двух квантовых чисел $n$ и $m$. В отличие от случая водородоподобного атома в постоянном магнитном поле, для которого задача является квазиточно решаемой, в случае магнитного поля $\mathbf{B}=(k / r) \mathbf{z}$ задача точно решаема. Легко проверить, что если нет внешнего магнитного поля, $K=0$, то восстанавливается энергетический спектр водородоподобного атома в отсутствие внешнего поля [1], [2]. 


\section{3. АТОМ В МАГНИТНОМ ПОЛЕ $\mathbf{B}=\left(k / r^{3}\right) \mathbf{z}$}

В кулоновской калибровке векторные потенциалы, связанные с магнитным полем $\mathbf{B}=\left(k / r^{3}\right) \mathbf{z}$, можно записать следующим образом:

$$
A_{x}=\frac{k \sin \theta}{r^{2}}, \quad A_{y}=-\frac{k \cos \theta}{r^{2}}, \quad A_{z}=0 .
$$

Гамильтониан водородоподобного атома в магнитном поле есть

$$
H_{2}=\frac{1}{2}\left(p^{2}+\frac{2 i K}{r^{3}} \partial_{\theta}+\frac{K^{2}}{r^{4}}\right)-\frac{Z}{r} .
$$

Мы полагаем, что собственная функция имеет вид

$$
\Phi(r, \theta)=\frac{u(r)}{\sqrt{r}} e^{i m \theta} .
$$

Радиальная волновая функция $u(r)$ подчиняется следующему дифференциальному уравнению второго порядка:

$$
\frac{1}{2}\left(-\frac{d^{2}}{d r^{2}}+\frac{m^{2}-1 / 4}{r^{2}}-\frac{2 K m}{r^{3}}+\frac{K^{2}}{r^{4}}-\frac{2 Z}{r}\right) u(r)=E_{2} u(r) .
$$

Пусть оператор $H_{2 r}$ имеет вид

$$
H_{2 r}=\frac{1}{2}\left(-\frac{d^{2}}{d r^{2}}+\frac{m^{2}-1 / 4}{r^{2}}-\frac{2 K m}{r^{3}}+\frac{K^{2}}{r^{4}}-\frac{2 Z}{r}\right) .
$$

Сконструируем гамильтониан того же вида, что и $H_{2 r}$, чтобы получить энергетический спектр. Предположим, что функция $W(x)$ в формуле (4) задается как

$$
W(x)=\frac{\alpha}{x}+\frac{\beta}{x^{2}}+\gamma .
$$

Тогда гамильтониан (4) принимает вид

$$
H_{2}^{\mathrm{c}}=\frac{1}{2}\left(-\frac{d^{2}}{d x^{2}}+\frac{\alpha^{2}+\alpha+2 \beta \gamma}{x^{2}}+\frac{2 \alpha \beta+2 \beta}{x^{3}}+\frac{\beta^{2}}{x^{4}}+\frac{2 \alpha \gamma}{x}+\gamma^{2}+v(x)\right), \quad x \geqslant 0,
$$

где $v(x)$ является потенциалом, подлежащим определению. Предположим, что собственная функция гамильтониана $H_{2}^{c}$ имеет вид

$$
\varphi_{N}^{\mathrm{c}}(x)=\sum_{i=0}^{N} a_{i} x^{i} \exp \left(-\int W(x) d x\right)=\sum_{i=0}^{N} a_{i} x^{i} x^{-\alpha} e^{-\gamma x+\beta / x} .
$$

Ясно, что должны существовать ограничения $\alpha<0, \beta<0, \gamma>0$, чтобы волновая функция была конечной. Подстановка $\varphi_{N}^{\mathrm{c}}(x)$ в уравнение Шредингера

$$
H_{2}^{\mathrm{c}} \varphi_{N}^{\mathrm{c}}(x)=E_{2}^{\mathrm{c}} \varphi_{N}^{\mathrm{c}}(x)
$$

дает

$$
v(x)=-\frac{2 \alpha a_{1}}{a_{0} x}+\frac{2 \beta a_{1}^{2}}{a_{0}^{2} x}-\frac{4 \beta a_{2}}{a_{0} x}-\frac{2 \beta a_{1}}{a_{0} x^{2}}, \quad E_{2}^{c}=0 .
$$


Рекуррентные соотношения для коэффициентов $a_{i}$ таковы:

$$
\begin{aligned}
\left(\frac{2 \alpha a_{1}}{a_{0}}\right. & \left.-\frac{2 \beta a_{1}^{2}}{a_{0}^{2}}+\frac{4 \beta a_{2}}{a_{0}}-2 \gamma(i+1)\right) a_{i+1}+ \\
& +\left(\frac{2 \beta a_{1}}{a_{0}}+(i+2)(i+1)-2 \alpha(i+2)\right) a_{i+2}-2 \beta(i+3) a_{i+3}=0 .
\end{aligned}
$$

Если $i=N-1$, то равенство (11) сводится к

$$
\frac{2 \alpha a_{1}}{a_{0}}-\frac{2 \beta a_{1}^{2}}{a_{0}^{2}}+\frac{4 \beta a_{2}}{a_{0}}=2 \gamma N .
$$

Комбинируя соотношения (10) и (12), непосредственно получаем

$$
\begin{aligned}
H_{2}^{\mathrm{c}}=\frac{1}{2}\left(-\frac{d^{2}}{d x^{2}}\right. & +\frac{\alpha^{2}+\alpha+2 \beta \gamma-2 \beta a_{1} / a_{0}}{x^{2}}+ \\
& \left.+\frac{2 \alpha \beta+2 \beta}{x^{3}}+\frac{\beta^{2}}{x^{4}}+\frac{2 \alpha \gamma-2 \gamma N}{x}+\gamma^{2}\right) .
\end{aligned}
$$

Пусть $H_{2}^{\mathrm{c}}$ имеет тот же вид, что и $H_{2 r}$; сравнение двух гамильтонианов дает

$$
\begin{gathered}
x=r, \quad \beta=-|K|, \quad \alpha=\left\{\begin{array}{rr}
-m-1, & K>0, \\
m-1, & K<0,
\end{array}\right. \\
\gamma=\frac{Z}{N+|m|+1}, \quad a_{1}=\frac{Z}{N+|m|+1}-\frac{1 / 4+|m|}{2 K},
\end{gathered}
$$

где мы полагаем, что $a_{0}=1$. Следует заметить, что значение $m$ ограничено в силу неравенства $\alpha<0$ : если магнитное поле направлено вдоль положительной части оси $z$, т. е. $K>0$, то $m \geqslant 0$; если магнитное поле направлено вдоль отрицательной части оси $z, K<0$, то $m \leqslant 0$. Следовательно, квантовые числа углового момента могут быть только неотрицательными или только неположительными целыми числами. Перепишем $\alpha$ как $\alpha=-|m|-1$.

Из соотношений (9), (13) и (14) получаем энергию водородоподобного атома:

$$
E_{N m}=-\frac{1}{2} \frac{Z^{2}}{(N+|m|+1)^{2}} .
$$

Кажется, что энергия определяется двумя квантовыми числами $N$ и $m$, и, таким образом, весь энергетический спектр можно получить, измененяя $N$ и $m$, но на самом деле это не так. Существуют два ограничения, наложенные на коэффициент $a_{1}$, одно из них вытекает из рекуррентного соотношения (11), а другое - из формулы для $a_{1}$ в (14). Оба ограничения выполняются, только если напряженность магнитного поля $k$ или заряд $Z$ рассматриваются как параметр: если фиксировано магнитное поле, то только определенные значения $Z$ разрешены при фиксированных $N$ и $m$; если заряд $Z$ фиксирован, только специальный набор магнитных полей разрешен. Ниже в качестве примера мы рассмотрим случай, когда в (5) порядок $N=1$. 
ПримеР 1. Если $N=1$, то $H_{2}^{\mathrm{c}}$ имеет вид

$$
\begin{aligned}
H_{2}^{\mathrm{c}}=\frac{1}{2}\left(-\frac{d^{2}}{d x^{2}}+\right. & \frac{\alpha^{2}+\alpha+2 \beta \gamma-2 \beta a_{1}}{x^{2}}+ \\
& \left.+\frac{2 \alpha \beta+2 \beta}{x^{3}}+\frac{\beta^{2}}{x^{4}}+\frac{2 \alpha \gamma-2 \gamma}{x}+\gamma^{2}\right) .
\end{aligned}
$$

Параметры этого выражения таковы:

$$
\alpha=-|m|-1, \quad \beta=-|K|, \quad \gamma=\frac{Z}{2+|m|}, \quad a_{1}=\frac{Z}{2+|m|}-\frac{1 / 4+|m|}{2|K|} .
$$

Рекуррентное соотношение (11) принимает вид

$$
\alpha a_{1}-\beta a_{1}^{2}-\gamma=0
$$

Формула для $a_{1}$ в $(15)$ и соотношение (16) выполняются, только если напряженность магнитного поля $K$ или заряд $Z$ рассматриваются как параметр:

$$
(-|m|-1)\left(\frac{Z}{2+|m|}-\frac{1 / 4+|m|}{2|K|}\right)+|K|\left(\frac{Z}{2+|m|}-\frac{1 / 4+|m|}{2 K}\right)^{2}-\frac{Z}{2+|m|}=0 .
$$

Если заряд $Z$ фиксирован, то разрешен только определенный набор магнитных полей,

$$
|K|=(|m|+2) \frac{2|m|+9 / 4+\sqrt{(|m|+2)^{2}+2(1 / 4+|m|)}}{2 Z},
$$

или, эквивалентно, если фиксировано внешнее магнитное поле, то имеется ограничение на значение $Z$. Из формул (7) и (13) мы получаем энергетический уровень и волновую функцию:

$$
\begin{gathered}
E_{2}=-\frac{1}{2} \frac{Z^{2}}{(|m|+2)^{2}}, \\
\psi(r, \theta)=\frac{U(r)}{\sqrt{r}} e^{i m \theta}=\left[1+\left(\frac{Z}{2+|m|}-\frac{1 / 4+|m|}{2|K|}\right) r\right] r^{m+1 / 2} \times \\
\times \exp \left(-\frac{Z}{2+|m|} r-\frac{|K|}{r}\right) e^{i m \theta} .
\end{gathered}
$$

\section{4. ЗАКЛЮЧЕНИЕ}

В настоящей статье обсуждаются задачи для плоского водородоподобного атома, движущегося в магнитных полях вида $\mathbf{B}=(k / r) \mathbf{z}$ и $\mathbf{B}=\left(k / r^{3}\right) \mathbf{z}$. Вместо непосредственного решения уравнения Шредингера мы построили гамильтониан, который имеет тот же вид, что и гамильтониан водородоподобного атома в однородных магнитных полях; сравнивая гамильтонианы, мы получили энергетический спектр. Результаты показали, что в случае магнитного поля $\mathbf{B}=(k / r) \mathbf{z}$ получается весь спектр, и вырождение энергии снимается. В отличие от известных результатов при отсутствии магнитного поля, в случае неоднородного магнитного поля квантовые числа углового момента могут не быть целыми числами, они определяются напряженностью магнитного поля и атомным зарядом. В случае магнитного поля $\mathbf{B}=\left(k / r^{3}\right) \mathbf{z}$ аналитические решения уравнения Шредингера возможны для определенных значений заряда $Z$ или напряженности магнитного поля $k$; таким образом, 
продемонстрирован физический пример квазиточно решаемой задачи. Следовательно, плоское движение электронов в неоднородных магнитных полях увеличивает круг физических моделей, которые описываются точно и квазиточно решаемыми задачами в квантовой механике.

Благодарности. Работа выполнена при финансовой поддержке NSF of China (гранты № 11247274, 11075115), а также при финансовой поддержке Fundamental Research Funds for the Central Universities (грант № 3122014K006).

\section{Список литературы}

[1] J. Zeng, Quantum Mechanics, Science Press, Beijing, 2000 (in Chinese).

[2] W. Greiner, Quantum Mechanics. An Introduction, Springer, Berlin, 1989.

[3] M. A. Shifman, Lie Algebras, Cohomology, and New Applications to Quantum Mechanics (Springfield, MO, March 20-21,1992), Contemporary Mathematics, 160, eds. N. Kamran, P. J. Olver, AMS, Providence, RI, 1994.

[4] M. A. Shifman, Internat. J. Modern Phys. A, 4:12 (1989), 2897-2952.

[5] B. A. Turbiner, Commun. Math. Phys., 118:3 (1988), 467-474.

[6] M. A. Shifman, ITEP Lectures on Particle Physics and Field Theory, World Scientific Lecture Notes in Physics, 62, World Sci., Singapore, 1999.

[7] A. G. Ushveridze, Quasi-Exactly Solvable Models in Quantum Mechanics, IOP, Bristol, 1994.

[8] A. V. Zabrodin, TMФ, 104:1 (1995), 8-24.

[9] С. М. Клишевич, ТМФ, 150:2 (2007), 237-248.

[10] A. Samanta, S. K. Ghosh, Phys. Rev. A, 42:3 (1990), 1178-1183.

[11] M. Taut, Phys. Rev. A, 48:5 (1993), 3561-3566.

[12] A. V. Turbiner, Phys. Rev. A, 50:6 (1994), 5335-5337, arXiv: hep-th/9406018.

[13] А. Д. Донков, В. Г. Кадышевский, М. Д. Матвеев, Р. М. Мир-Касимов, ТМФ, 8:1 (1971), $61-72$.

[14] Y. Brihaye, P. Kosinski, Modern Phys. Lett. A, 13:18 (1998), 1445-1452.

[15] P.-F. Loos, P. M. W. Gill, Phys. Rev. Lett., 103:12 (2009), 123008, 4 pp.

[16] A. H. MacDonald, D. S. Ritchie, Phys. Rev. B, 33:12 (1986), 8336-8344.

[17] P. Martin, J. J. Rodriguez-Nuñez, J. L. Marquez, Phys. Rev. B, 45:15 (1992), 8359-8362.

[18] H. Friedrich, D. Wintgen, Phys. Rep., 183:2 (1989), 37-79.

[19] W. Rösner, H. Herold, H. Ruder, G. Wunner, Phys. Rev. A, 28:4 (1983), 2071-2077.

[20] M. Taut, J. Phys. A, 27 (1994), 1045.

[21] M. Taut, J. Phys. A, 28:7 (1995), 2081-2085.

[22] M. Taut, J. Phys. A: Math. Gen., 32:29 (1999), 5509-5515.

[23] V. M. Villalba, R. Pino, Phys. Lett. A, 238:1 (1998), 49-53, arXiv: cond-mat/9712044.

[24] C.-L. Ho, V.R. Khalilov, Phys. Rev. A, 61:3 (2000), 032104, 7 pp., arXiv: quant-ph/0003086.

[25] C.-M. Chiang, C.-L. Ho, Phys. Rev. A, 63:6 (2001), 062105, 5 pp., arXiv: hep-th/0011005. [26] C.-L. Ho, P. Roy, Ann. Phys., 312:1 (2004), 161-176. 